International Background Paper

Wealth Tax Commission

\title{
Wealth taxes in foreign countries
}

Author

Emma Chamberlain OF ECONOMICS AND POLITICAL SCIENCE —

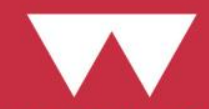
WARWICK IVK 


\section{WEALTH TAXES IN FOREIGN COUNTRIES}

Emma Chamberlain, Pump Court Tax Chambers

Wealth Tax Commission Background Paper no. 130

Published by the Wealth Tax Commission

www.ukwealth.tax 


\section{Acknowledgements}

The Wealth Tax Commission acknowledges funding from the Economic and Social Research Council (ESRC) through the CAGE at Warwick (ES/L011719/1) and a COVID-19 Rapid Response Grant (ES/V012657/1), and a grant from Atlantic Fellows for Social and Economic Equity's COVID-19 Rapid Response Fund. 


\begin{tabular}{|c|c|c|c|c|c|c|c|c|c|}
\hline Countries & History & Assets taxed & $\begin{array}{c}\text { Tax base } \\
\text { Household/ } \\
\text { individual }\end{array}$ & Territoritality & Trusts/Foundations & $\begin{array}{l}\text { Valuation issues/debt } \\
\text { Liquidity concerns }\end{array}$ & $\begin{array}{l}\text { Interaction with } \\
\text { other taxes }\end{array}$ & $\begin{array}{l}\text { Enforcement/assessment } \\
\text { /collection costs }\end{array}$ & $\begin{array}{c}\text { Yield/ } \\
\text { Rates/Exempt } \\
\text { Thresholds } \\
\end{array}$ \\
\hline France & $\begin{array}{l}\text { Introduced in } 1945 \text { as } \\
\text { an exceptional tax to } \\
\text { finance war } \\
\text { reparations and } \\
\text { became permanent as } \\
\text { IGF ('Iimpot sur les } \\
\text { grandes fortunes). } \\
\text { In } 1989 \text { was renamed } \\
\text { ISF or solidarity tax; } \\
\text { ISF was abolished } \\
2017 . \\
2018 \text { - Replaced by } \\
\text { IFI: mechanism almost } \\
\text { identical but now } \\
\text { solely levied annually } \\
\text { on real estate. } \\
\text { Allegedly } 70 \% \text { French } \\
\text { want return of full ISF. }\end{array}$ & $\begin{array}{l}\text { Since 2018 WT } \\
\text { only charged on } \\
\text { real estate: } \\
\text { includes shares } \\
\text { attributable to } \\
\text { land where land } \\
\text { not used in } \\
\text { trading business; } \\
\text { life insurance } \\
\text { contracts } \\
\text { invested in real } \\
\text { estate. } \\
\text { Some minority } \\
\text { interests in } \\
\text { companies } \\
\text { holding land are } \\
\text { exempt if } \\
\text { broadly less than } \\
10 \% \text { or where } \\
\text { the collective } \\
\text { investment holds } \\
\text { less than } 20 \% \text { of } \\
\text { taxable real } \\
\text { estate asset. } \\
\text { Real estate } \\
\text { exempt if linked } \\
\text { with taxpayer's } \\
\text { main activity or } \\
\text { used by company } \\
\text { where he works. } \\
\text { Until } 2018 \text { - ISF } \\
\text { was on all assets } \\
\text { although } \\
\text { antiques, art, } \\
\text { intellectual, } \\
\text { property rights, } \\
\text { pensions, } \\
\text { woodlands and } \\
\text { 'assets needed } \\
\text { for taxpayer's } \\
\text { main } \\
\text { professional } \\
\text { activity' or a in } \\
\text { company where } \\
\text { a taxpayer or } \\
\text { director or } \\
\text { mainly works } \\
\text { were exempt. }\end{array}$ & $\begin{array}{l}\text { Payable by } \\
\text { households } \\
\text { whose total } \\
\text { netr real estate } \\
\text { (formerly all } \\
\text { assets) } \\
\text { exceed } € 1.3 \mathrm{~m} \\
\text { on } 1 \text { Jan each } \\
\text { year. } \\
\text { Spouses' and } \\
\text { co-habitees' } \\
\text { and minor } \\
\text { children's } \\
\text { wealth is } \\
\text { combined and } \\
\text { no increase in } \\
\text { exempt } \\
\text { threshold } \\
\text { given. }\end{array}$ & $\begin{array}{l}\text { Worldwide real } \\
\text { estate } \\
\text { (formerly all) } \\
\text { taxed on } \\
\text { French } \\
\text { residents if } \\
\text { French resident } \\
\text { for more than } 5 \\
\text { years. } \\
\text { Otherwise } \\
\text { limited to } \\
\text { French real } \\
\text { estate (pre- } \\
2018 \text { all French } \\
\text { assets) for non- } \\
\text { residents. } \\
\\
\text { No exit wealth } \\
\text { tax levied on } \\
\text { departure. } \\
\text { Treaty } \\
\text { exemption } \\
\text { between } \\
\text { France and Gulf } \\
\text { countries } \\
\text { providing } \\
\text { exemption } \\
\text { from IFI for } \\
\text { Gulf residents } \\
\text { on French real } \\
\text { estate provided } \\
\text { minimum } \\
\text { investment in } \\
\text { French } \\
\text { securities. }\end{array}$ & $\begin{array}{l}\text { Pension and charitable } \\
\text { trusts exempt. } \\
\text { Whatever type of trust is } \\
\text { used, the French resident } \\
\text { settlor is taxed on all } \\
\text { trust assets (now limited } \\
\text { to worldwide real estate } \\
\text { held in trust). } \\
\text { If non-resident settlor, } \\
\text { he pays on French real } \\
\text { estate owned by trust as } \\
\text { if assets owned } \\
\text { personally. } \\
\text { Trustees pay at highest } \\
1.5 \% \text { rate without } € 1.3 m \\
\text { exemption if settlor does } \\
\text { not pay and trust has } \\
\text { French connection. } \\
\text { On death of settlor } \\
\text { French resident } \\
\text { beneficiaries taxed by } \\
\text { rebuttable presumption } \\
\text { on their share of assets } \\
\text { unless can show that } \\
\text { trust assets do not } \\
\text { confer any financial } \\
\text { capacity. If beneficiaries } \\
\text { do not pay then trustees } \\
\text { pay at highest marginal } \\
\text { rate of } 1.5 \% / n o ~ € 1.3 m \\
\text { exemption. } \\
\text { Trustees must file a } \\
\text { return each year if any } \\
\text { French resident } \\
\text { beneficiaries or French } \\
\text { settlor or French real } \\
\text { estate. } \\
\text { Private foundations } \\
\text { treated as trusts. } \\
\text { Usufructs - person with } \\
\text { bure interest in land does } \\
\text { not pay WT but } \\
\text { usufructuary does. }\end{array}$ & $\begin{array}{l}\text { Annual valuations needed. } \\
30 \% \text { discount given on } \\
\text { main residence. } \\
\text { From } 2018 \text { IFI - no } \\
\text { deduction for connected } \\
\text { party debts and various } \\
\text { anti-avoidance provisions } \\
\text { on debt deduction } \\
\text { generally. Must be } \\
\text { incurred for the property } \\
\text { purchase or improvement. } \\
\text { Some max limits imposed. } \\
\text { Until } 2018 \text { - Under ISF to } \\
\text { encourage investment in } \\
\text { SMEs a reduction was } \\
\text { given of } 50 \% \text { of capital } \\
\text { increase up to a limit of } \\
\text { circa €50,000 if held SME } \\
\text { for } 5 \text { years. } \\
\text { Capping mechanism largely } \\
\text { dealt with liquidity issues } \\
\text { for ISF. Only available to } \\
\text { French residents. } \\
\text { Cannot elect to defer } \\
\text { payment. }\end{array}$ & $\begin{array}{l}\text { Capping } \\
\text { mechanism - } \\
\text { wealth tax and } \\
\text { income tax due } \\
\text { from the same } \\
\text { household } \\
\text { cannot exceed } \\
75 \% \text { of the } \\
\text { previous year's } \\
\text { household } \\
\text { income. Only } \\
\text { available for } \\
\text { French residents } \\
\text { - still there for } \\
\text { IFl. } \\
\text { IFI deductible } \\
\text { from inheritance } \\
\text { taxif due but not } \\
\text { yet paid by time } \\
\text { of death. } \\
\text { 75\% of gifts to } \\
\text { charities are } \\
\text { deductible } \\
\text { against WT up to } \\
\text { annual limit of } \\
\text { €50,000. } \\
\text { Other taxes } \\
\text { include IHT } \\
\text { Taxes Fonciere } \\
\text { on property and } \\
\text { CGT. WT not } \\
\text { deductible. }\end{array}$ & $\begin{array}{l}\text { WT filed as part of } \\
\text { income tax returns. } \\
\text { Trustees holding French } \\
\text { real estate have to file } \\
\text { annual returns revealing } \\
\text { identify of settlor and } \\
\text { beneficiaries and pay } \\
\text { higher } 1.5 \% \text { if settlor or } \\
\text { beneficiaries do not pay. } \\
\text { Company holding French } \\
\text { real estate has to pay } 3 \% \\
\text { of gross market value } \\
\text { unless files specific } \\
\text { return revealing the } \\
\text { identity of shareholders } \\
\text { or trust. } \\
\text { From } 2018 \text { avoidance } \\
\text { occurs round splitting } \\
\text { real estate assets with } \\
\text { adult children, use of } \\
\text { debt and capping } \\
\text { mechanism even under } \\
\text { IFI. Also ensuring real } \\
\text { estate used by family } \\
\text { business. } \\
\text { Until } 2018 \text { - under ISF } \\
\text { manipulation occurred } \\
\text { round business } \\
\text { exemptions, } \\
\text { accumulation of income } \\
\text { in companies that were } \\
\text { exempt. } \\
\text { Abuse of rights doctrine } \\
\text { gives some ability to } \\
\text { make tax adjustments } \\
\text { but rarely operates. } \\
\text { Collection costs } € 103 m \\
\text { in } 2016 \text { on ISF - about } \\
0.78 \% \text { of total tax } \\
\text { revenue. } 351,152 \text { wealth } \\
\text { tax returns in } 2016 .\end{array}$ & $\begin{array}{l}\text { ISF raised } € 5.1 \mathrm{bn} \text { in } \\
2017 ; \text {; IFI raised E2.1bn } \\
\text { in 2019. } \\
\text { ISF/IFI levied on } \\
\text { households with net } \\
\text { wealth above } 1 \text { 1.3m. } \\
\text { When } € 1.3 \mathrm{~m} \text { threshold } \\
\text { reached, WT becomes } \\
\text { due as from } € 800,000 \\
\text { Progressive rates from } \\
0.5 \% \text { up to } 1.5 \% \text { over } \\
€ 10 \mathrm{~m} \text {. } \\
\text { Not banded rates and } \\
\text { no cap by reference to } \\
\text { wealth only by income. } \\
\text { Same rates operate for } \\
\text { IFI except only levied } \\
\text { on land or shares } \\
\text { whose value is } \\
\text { attributable to land e.g. } \\
\text { owning a house worth } \\
€ 20 \mathrm{~m} \text { now results in } \\
€ 244,467 \text { House of } \\
€ 2 \mathrm{~m} \text { results in } 7438 \\
\text { IFI. }\end{array}$ \\
\hline
\end{tabular}




\begin{tabular}{|c|c|c|c|c|c|c|c|c|c|}
\hline Countries & History & Assets taxed & $\begin{array}{c}\text { Tax base } \\
\text { Household/ } \\
\text { individual }\end{array}$ & Territoritality & Trusts/Foundations & $\begin{array}{l}\text { Valuation issues/debt } \\
\text { Liquidity concerns }\end{array}$ & $\begin{array}{l}\text { Interaction with } \\
\text { other taxes }\end{array}$ & $\begin{array}{l}\text { Enforcement/assessment } \\
\text { /collection costs }\end{array}$ & $\begin{array}{c}\text { Yield/ } \\
\text { Rates/Exempt } \\
\text { Thresholds } \\
\end{array}$ \\
\hline $\begin{array}{l}\text { Germany } \\
\text { (Gy) }\end{array}$ & $\begin{array}{l}\text { Abolished in December } \\
1996 . \\
\text { Long history of WTs in } \\
\text { Gy since early Middle } \\
\text { Ages. } \\
1919 \text { one-off net } \\
\text { wealth tax to pay for } \\
\text { Versailles Treaty costs. } \\
1922 \text { First National } \\
\text { Regular Annual Wealth } \\
\text { Tax introduced. } \\
1952 \text { - one-off WT of } \\
50 \% \text { payable by } \\
\text { instalments until } 1979 . \\
\text { No wealth tax charged } \\
\text { in Eastern Germany } \\
\text { after Reunification. } \\
\text { From Jan } 1997 \text { - } \\
\text { abolished as in } 1995 \text { it } \\
\text { was held } \\
\text { unconstitutional as } \\
\text { valuation massively } \\
\text { preferred immovable } \\
\text { property over shares. }\end{array}$ & $\begin{array}{l}\text { All assets with } \\
\text { few exemptions. } \\
\text { Pensions } \\
\text { exempt. } \\
\text { Significant } \\
\text { discount on real } \\
\text { estate. }\end{array}$ & $\begin{array}{l}\text { Tax } \\
\text { allowance, } \\
\text { single, DM } \\
120,000, \\
\text { married } \\
\text { couples DM } \\
240,000 \text { and } \\
\text { each child } \\
\text { given an } \\
\text { allowance of } \\
\text { DM 120,000. } \\
\text { Additional } \\
\text { allowances } \\
\text { for over } 60 \text { or } \\
\text { disabled. } \\
\text { Companies } \\
\text { also taxed } \\
\text { (not dealt } \\
\text { with here). }\end{array}$ & $\begin{array}{l}\text { WT on German } \\
\text { residents or on } \\
\text { non-residents } \\
\text { with German } \\
\text { assets of any } \\
\text { sort. } \\
\text { Tail - liability } \\
\text { for } 10 \text { years } \\
\text { after leaving } \\
\text { (with reduction } \\
\text { after 5) esp. } \\
\text { where } \\
\text { individual } \\
\text { moved to a } \\
\text { foreign } \\
\text { jurisdiction } \\
\text { with lower } \\
\text { taxes on } \\
\text { income but } \\
\text { with substantial } \\
\text { economic } \\
\text { interests in Gy. }\end{array}$ & $\begin{array}{l}\text { If settlor and any } \\
\text { member of family (widely } \\
\text { defined) were entitled to } \\
\text { more than half of income } \\
\text { or more than half of } \\
\text { economic ownership } \\
\text { wealth and income } \\
\text { attributed transparently } \\
\text { to the settlor or } \\
\text { beneficiaries. }\end{array}$ & $\begin{array}{l}\text { Real estate generally had } \\
\text { low historic valuations. } \\
1990 \text { s -represented } 6- \\
30 \% \text { of fair market value. } \\
\text { Businesses assessed every } \\
3 \text { years. Included claims } \\
\text { against a pension fund but } \\
\text { excluded claims against a } \\
\text { work-related pension fund } \\
\text { or after the taxpayer } \\
\text { turned } 60 \text {. } \\
\text { Jewellery over } € 5000 \text {; art } \\
\text { and collections over } \\
\text { €10,000 provided the } \\
\text { artist was dead. } \\
\text { Debt deductible including } \\
\text { debt borrowings to pay the } \\
\text { WT itself. } \\
\text { Could defer payment } \\
\text { under hardship clause } \\
\text { which had high threshold. }\end{array}$ & $\begin{array}{l}\text { No cap by } \\
\text { reference to } \\
\text { total income. } \\
\text { Foreign WTs } \\
\text { creditable } \\
\text { against German } \\
\text { WT if WT double } \\
\text { taxation treaty. }\end{array}$ & \begin{tabular}{|l|} 
Annual tax reassessed \\
every 3 years. \\
\\
Collection costs disputed \\
- Federal Govt estimated \\
$4-4.5 \%$ of WT revenue \\
but some estimates are \\
much higher and \\
probably above average \\
cost of other taxes.. \\
\\
Prone to underreporting. \\
Could move assets into \\
foreign establishments \\
and try and get treaty \\
relief.
\end{tabular} & $\begin{array}{l}\text { In } 1996 \text { - raised DM } \\
7.8 \mathrm{bn}-=€ 4 \mathrm{bn} .1 \% \text { of } \\
\text { entire tax revenue or } \\
0.2 \% \text { of GDP } \\
\text { No cap by wealth or } \\
\text { income. } \\
\text { Annual flat rate of } 1 \% \\
\text { except for forestry or } \\
\text { agriculture when rate } \\
\text { was } 0.5 \% \text {. } \\
\text { Exempt allowance of } \\
€ 60,000 \text {. Married } \\
\text { couples had } € 120,000 \\
\text { and for each minor } \\
\text { child an alllowance of } \\
\text { €60,000 (then } \\
\text { DM120,000) was } \\
\text { added. An additional } \\
\text { allowance of } € 25,000 \\
\text { was granted when the } \\
\text { taxpayer was over } 60 \\
\text { or disabled. } \\
€ 50,000 \text { allowance } \\
\text { granted for entities } \\
\text { engaged in agriculture } \\
\text { or forestry. }\end{array}$ \\
\hline Italy & $\begin{array}{l}\text { Forced levy in } 1992 . \\
\text { One-off tax of } 0.6 \% \\
\text { was levied in } 1992 \text { on } \\
\text { value of Italian bank } \\
\text { deposits irrespective } \\
\text { of tax residence. } \\
\text { Backdated by } 2 \text { days to } \\
\text { capture possible } \\
\text { withdrawals. } \\
1992 \text { - also introduced } \\
\text { wealth tax on Italian } \\
\text { land which still exists } \\
\text { today known as IIIU } \\
\text { on Italian property and } \\
\text { IVIE on foreign real } \\
\text { estate. }\end{array}$ & $\begin{array}{l}\text { Tax on real } \\
\text { estate located } \\
\text { abroad and in } \\
\text { Italy but with } \\
\text { main residence } \\
\text { exempt unless } \\
\text { luxury property. } \\
\text { Stamp duty like } \\
\text { tax on financial } \\
\text { investments not } \\
\text { discussed } \\
\text { further here - } \\
\text { see paper. }\end{array}$ & $\begin{array}{l}\text { Tax liability } \\
\text { per property } \\
\text { split between } \\
\text { couples but } \\
\text { no difference } \\
\text { in rate or } \\
\text { exempt } \\
\text { threshold as } \\
\text { levied per } \\
\text { property. }\end{array}$ & $\begin{array}{l}\text { All persons pay } \\
\text { on Italian real } \\
\text { estate and } \\
\text { Italian } \\
\text { residents pay } \\
\text { IVIE on foreign } \\
\text { property unless } \\
\text { within the } \\
\text { special Art } 24 \\
\text { bis regime. }\end{array}$ & $\begin{array}{l}\text { Italian resident } \\
\text { trusts - pay IVIE and } \\
\text { IVAFE on foreign assets } \\
\text { from } 2020 \text {. } \\
\text { Non-resident trusts pay } \\
\text { IMU on Italian real estate } \\
\text { and Italian financial } \\
\text { investments. }\end{array}$ & $\begin{array}{l}\text { Cadastral value for Italian } \\
\text { and equivalent for foreign } \\
\text { property -much lower than } \\
\text { market value. } \\
\text { Levied on gross not net } \\
\text { values. } \\
\text { No liquidity concerns. A } \\
€ 500,000 \text { Italian property } \\
\text { will suffer } € 3800 \text { pa tax. A } \\
€ 20 \mathrm{~m} \text { property will suffer } \\
€ 152,500 \text { pa tax. }\end{array}$ & $\begin{array}{l}\text { No deductibility } \\
\text { against } \\
\text { inheritance and } \\
\text { gift taxes but } \\
\text { latter is low } \\
\text { anyway and } \\
\text { imposed at } \\
\text { maximum } 8 \% \text { - } \\
\text { usually 4\%. }\end{array}$ & $\begin{array}{l}\text { Loophole amended to } \\
\text { include Italian trusts that } \\
\text { hold foreign financial } \\
\text { assets or foreign real } \\
\text { estate. Previously } \\
\text { exemption was possible. } \\
\text { General anti-abuse } \\
\text { provision. }\end{array}$ & $\begin{array}{l}\text { Rates between } 0.76 \% \text { - } \\
-0.1 \% \text { for IMU } \\
\text { depending on type of } \\
\text { building. } 0.2 \% \text { for IVIE } \\
\text { (foreign estate). } \\
\\
\text { De minimis exemption } \\
\text { threshold of } € 26,300 \\
\text { on foreign property. } \\
\text { No minimal exemption } \\
\text { for Italian property - } \\
\text { does not operate by } \\
\text { reference to bands } \\
\text { with an overall tax cap } \\
\text { but at a low flat rate on } \\
\text { the entire value. }\end{array}$ \\
\hline
\end{tabular}




\begin{tabular}{|c|c|c|c|c|c|c|c|c|c|}
\hline India & $\begin{array}{l}\text { WT from 1957-2015. } \\
\text { Replaced with an } \\
\text { additional } 2 \% \\
\text { surcharge on the } \\
\text { superrich with high } \\
\text { taxable income. }\end{array}$ & $\begin{array}{l}\text { Before 1993- } \\
\text { broadly all assets } \\
\text { except for } \\
\text { agricultural land } \\
\text { and pensions. } \\
\\
\text { After } 1993 \text { only } \\
\text { on houses } \\
\text { (commercial or } \\
\text { residential other } \\
\text { than main } \\
\text { residence, } \\
\text { certain rental } \\
\text { properties), cars } \\
\text { yachts, cash over } \\
\text { 50,000 rupees. }\end{array}$ & $\begin{array}{l}\text { Levied on } \\
\text { wealth per } \\
\text { individual not } \\
\text { household but } \\
\text { wealth of } \\
\text { spouses and } \\
\text { minor } \\
\text { children } \\
\text { included in } \\
\text { tax base of } \\
\text { same } \\
\text { individual } \\
\text { (spousal } \\
\text { wealth only } \\
\text { included if } \\
\text { donor } \\
\text { individual had } \\
\text { gifted asset to } \\
\text { spouse for } \\
\text { less than } \\
\text { market value) }\end{array}$ & $\begin{array}{l}\text { Non-residents } \\
\text { paid on any } \\
\text { Indian assets } \\
\text { including } \\
\text { enveloped } \\
\text { vehicles } \\
\text { holding Indian } \\
\text { land. } \\
\text { Exemption on } \\
\text { assets located } \\
\text { outside India } \\
\text { for first } 3 \text { years } \\
\text { after arriving in } \\
\text { India. No exit } \\
\text { tax as such but } \\
\text { typically a tail } \\
\text { of } 4 \text { years } \\
\text { operated from } \\
\text { exit for the WT } \\
\text { to apply. }\end{array}$ & $\begin{array}{l}\text { Public and charitable } \\
\text { trusts exempt. } \\
\text { All trusts taxed where } \\
\text { Indian assets or Indian } \\
\text { resident beneficiaries } \\
\text { and status of settlor } \\
\text { irrelevant. }\end{array}$ & $\begin{array}{l}\text { Annual valuations. } \\
\text { Special rules for land, } \\
\text { jewellery partnerships. } \\
\text { Main residence exempt. } \\
\text { All agricultural assets } \\
\text { excluded. Discretion on } \\
\text { paying yy instalments or } \\
\text { waiving interest on } \\
\text { instalments to deal with } \\
\text { liquidity. } \\
\text { Debt is deductible. }\end{array}$ & $\begin{array}{l}\text { Inheritance tax } \\
\text { previously rates } \\
\text { up to } 85 \% \text {. } \\
\text { No deduction for } \\
\text { WT allowed } \\
\text { against other } \\
\text { taxes. No cap } \\
\text { against income } \\
\text { operated. }\end{array}$ & $\begin{array}{l}\text { Attempts to get within } \\
\text { exempt categories. } \\
\text { Large scale evasion. } \\
\text { Self-assessment annually } \\
\text { by separate regime. } \\
\text { Lengthy disputes over } \\
\text { valuation. }\end{array}$ & $\begin{array}{l}\text { Raised } 10.8 \text { million } \\
\text { rupees in } 2014 / 15 \\
\text { From } 1993 \text { ceased to } \\
\text { be progressive and } \\
\text { levied at flat rate of } 1 \% \\
\text { on net wealth above } \\
\text { specified threshold } \\
\text { which was } 3 \text { million } \\
\text { rupees (about } \\
£ 31,331 \text { ) in 2010. } \\
\text { No cap or banding } \\
\text { operated either by } \\
\text { reference to wealth or } \\
\text { income. }\end{array}$ \\
\hline Norway & $\begin{array}{l}\text { WT in force since } \\
1892 . \\
\text { Conservatives have } \\
\text { reduced rates since } \\
2014 \text { from } 1.1 \% \text { to } \\
0.85 \% \text { and certain } \\
\text { valuation discounts on } \\
\text { companies have } \\
\text { increased. Wealth tax } \\
\text { yield has increased. }\end{array}$ & $\begin{array}{l}\text { Most assets } \\
\text { taxed but with } \\
\text { discounts for } \\
\text { property. } \\
\text { Exemption for } \\
\text { pensions, crops, } \\
\text { goodwill rights } \\
\text { to creative } \\
\text { works. } \\
\text { Company shares } \\
\text { taxes on } \\
\text { shareholder. }\end{array}$ & $\begin{array}{l}\text { Wealth of } \\
\text { spouses and } \\
\text { minor } \\
\text { children } \\
\text { combined } \\
\text { with tax } \\
\text { exemption } \\
\text { doubled for } \\
\text { couples. } \\
\text { Cohabitees } \\
\text { not included. }\end{array}$ & $\begin{array}{l}\text { All Norwegian } \\
\text { residents taxed } \\
\text { from first year } \\
\text { of residence on } \\
\text { worldwide } \\
\text { assets. } \\
\text { Non-residents } \\
\text { pay WT only on } \\
\text { Norwegian real } \\
\text { estate. } \\
\text { However, to } \\
\text { become non- } \\
\text { resident and } \\
\text { avoid WT in } \\
\text { first three years } \\
\text { after leaving } \\
\text { you must give } \\
\text { up your } \\
\text { dwelling in } \\
\text { Norway and } \\
\text { spend less than } \\
\text { 62 days each in } \\
\text { Norway. }\end{array}$ & $\begin{array}{l}\text { Life interest trusts - } \\
\text { broadly the life tenant is } \\
\text { taxed on the value of the } \\
\text { asset. } \\
\text { Revocable trusts - trusts } \\
\text { disregarded and taxed on } \\
\text { settlor if power to } \\
\text { revoke. } \\
\text { Discretionary trusts - } \\
\text { Ptarmigan trust case } \\
\text { Olsen v Norway - } \\
\text { discretionary } \\
\text { beneficiaries with no } \\
\text { control influence or } \\
\text { benefit not subject to } \\
\text { WT. } \\
\text { WT lower for family } \\
\text { foundations. O.15\% } \\
\text { Beneficiaries only taxed } \\
\text { on actual distributions. }\end{array}$ & $\begin{array}{l}\text { Listed shares valued at } \\
\text { discount of } 65 \% \text { of listed } \\
\text { price. } \\
\text { Unlisted shares valued at } \\
65 \% \text { of market value with } \\
\text { proposed increase in } \\
\text { discount to } 55 \% \text { of market } \\
\text { value for } 2021 . \\
75 \% \text { discount for primary } \\
\text { residences, holiday homes } \\
\text { and } 10 \% \text { discount for } \\
\text { secondary residences. } 75 \% \\
\text { discount to be capped from } \\
2021 \text { for main residence of } \\
\text { up to } € 150,000 \text { in value } \\
\text { and then a } 50 \% \text { discount } \\
\text { will operate. } 35 \% \text { discount } \\
\text { for commercial property } \\
\text { rising to } 45 \% \text { in } 2021 . \\
\text { Taxpayers with a loss in } \\
2020 \text { due to COVID may } \\
\text { request deferred payment } \\
\text { of WT for one year. }\end{array}$ & $\begin{array}{l}\text { Inheritance } \\
\text { tax/gift tax } \\
\text { abolished in } \\
2014 . \\
\\
\text { WT not } \\
\text { deductible } \\
\text { against any } \\
\text { other tax. } \\
\text { Some tax treaty } \\
\text { relief against } \\
\text { other countries } \\
\text { with WT. }\end{array}$ & $\begin{array}{l}\text { Self-assessment regime } \\
\text { and WT assessed } \\
\text { annually. Advance } \\
\text { payment each quarter. } \\
\text { In } 2019 \text { some loopholes } \\
\text { closed. GAAR provision } \\
\text { operates but not } \\
\text { commonly used in WT. }\end{array}$ & $\begin{array}{l}0.85 \% \text { rate in } 2020 \text { on } \\
\text { net wealth over } \\
€ 150,000 \text { Reduced } \\
\text { from } 1.1 \% \text { recently. } \\
\text { Raised approx. } 11.6 \\
\text { billion }=1.1 \% \text { of total } \\
\text { tax revenue. } \\
\\
\text { No cap on the WT and } \\
\text { does not operate by } \\
\text { bands. }\end{array}$ \\
\hline
\end{tabular}




\begin{tabular}{|c|c|c|c|c|c|c|c|c|c|}
\hline Spain & $\begin{array}{l}\text { Introduced in 1977-as } \\
\text { a temporary measure. } \\
\text { Remained until 1992 } \\
\text { when Wealth Tax Law } \\
\text { enacted. } \\
2008-11 \text { WT } \\
\text { abolished. } \\
2011 \text { - reintroduced } \\
\text { initially temporarily } \\
\text { but annually extended } \\
\text { and in force today. } \\
\text { Suggestions that a } \\
\text { higher fortune tax } \\
\text { should be introduced. }\end{array}$ & $\begin{array}{l}\text { Exemption for } \\
\text { pensions, some } \\
\text { art works up to a } \\
\text { maximum } \\
€ 90,000, \\
\text { antiques, } \\
\text { household items, } \\
\text { intellectual } \\
\text { property or } \\
\text { creator } \\
\text { insurance } \\
\text { policies, some } \\
\text { active family } \\
\text { companies. } \\
\text { €300,000 } \\
\text { exemption for } \\
\text { main home for } \\
\text { Spanish } \\
\text { residents only. } \\
\text { Important WT } \\
\text { exemption for } \\
\text { small and } \\
\text { medium size } \\
\text { companies: } \\
\text { taxpayer must } \\
\text { own at least } 5 \% \\
\text { of shares or } 20 \% \\
\text { with connected } \\
\text { family members. } \\
\text { Taxpayer must } \\
\text { manage the } \\
\text { company and } \\
\text { receive salary } \\
\text { that represents } \\
\text { more than } 50 \% \\
\text { of his total } \\
\text { income derived } \\
\text { from any } \\
\text { business, } \\
\text { profession or } \\
\text { labour. } \\
\text { Company must } \\
\text { be trading. }\end{array}$ & $\begin{array}{l}\text { Levied } \\
\text { individually } \\
\text { and not on } \\
\text { family. } \\
\text { Each minor } \\
\text { child and each } \\
\text { parent has a } \\
€ 700,000 \\
\text { exemption. }\end{array}$ & $\begin{array}{l}\text { Non-resident } \\
\text { individuals pay } \\
\text { on all Spanish } \\
\text { assets not just } \\
\text { real estate but } \\
\text { not necessarily } \\
\text { on foreign } \\
\text { companies } \\
\text { Spanish real } \\
\text { estate or } \\
\text { Spanish estates. } \\
\text { No capping } \\
\text { mechanism } \\
\text { described } \\
\text { elsewhere } \\
\text { available for } \\
\text { non-residents. } \\
\text { Residents pay } \\
\text { on worldwide } \\
\text { assets from } \\
\text { date of arrival } \\
\text { in Spain unless } \\
\text { treaty resident } \\
\text { elsewhere. } \\
\text { However, } \\
\text { Beckhams law } \\
\text { regime means } \\
\text { immigrants are } \\
\text { taxed as non- } \\
\text { residents for } 6 \\
\text { years if they are } \\
\text { working in a } \\
\text { company not } \\
\text { controlled by } \\
\text { them. } \\
\text { Exit tax } \\
\text { imposed on } \\
\text { gains on } \\
\text { departure for } \\
\text { long term } \\
\text { Spanish } \\
\text { residents. No } \\
\text { exit tax for WT } \\
\text { purposes. }\end{array}$ & $\begin{array}{l}\text { Settlor or beneficiary } \\
\text { taxed. } \\
\text { While settlor is alive and } \\
\text { non-resident and assets } \\
\text { pass to beneficiaries only } \\
\text { on death, only Spanish } \\
\text { trust assets are taxed. } \\
\text { Spanish resident } \\
\text { beneficiaries can be } \\
\text { taxed earlier than } \\
\text { settlor's death if it is } \\
\text { inferred they get } \\
\text { benefits only. }\end{array}$ & $\begin{array}{l}\text { Annual valuation on } 31 \\
\text { December. } \\
\text { Real estate valued at } \\
\text { higher of acquisition value, } \\
\text { Cadastral value (usually } \\
\text { low) or revenue } \\
\text { assessment in a tax } \\
\text { proceeding. } \\
\text { Bank deposits - higher of } \\
31 \text { December amount or } \\
\text { average balance of last } \\
\text { quarter. } \\
\text { Loans - nominal value with } \\
\text { no account taken of } \\
\text { accrued interest. } \\
\text { Non-listed shares - net } \\
\text { book value where audited. } \\
\text { Otherwise higher value can } \\
\text { be taken based on average } \\
\text { profits or net book value or } \\
\text { share capital. } \\
\text { Life insurance - value } \\
\text { taken for WT purposes is } \\
\text { surrender value. } \\
\text { Jewellery and cars, non- } \\
\text { exempt art - market value. } \\
\text { Capping: WT and income } \\
\text { tax cannot exceed } 60 \% \text { of } \\
\text { taxable base for Spanish } \\
\text { residents but only } 80 \% \text { of } \\
\text { WT may be reduced } \\
\text { whatever the level of } \\
\text { income. } \\
\text { Debt - principal but not } \\
\text { interest is deductible. } \\
\text { No deduction for debt for } \\
\text { non-residents unless } \\
\text { borrowings taken out to } \\
\text { purchase Spanish assets } \\
\text { not exempt from WT. }\end{array}$ & $\begin{array}{l}\text { Regions have } \\
\text { very different } \\
\text { deductions. } \\
\text { Inheritance and } \\
\text { gift tax also } \\
\text { applies and not } \\
\text { deductible - } \\
\text { rates between } \\
7.65 \% \text { to } 34 \% . \\
95 \% \text { exemption } \\
\text { applies to active } \\
\text { family trading } \\
\text { companies. } \\
\text { Different regions } \\
\text { vary enormously } \\
\text { e.g. Madrid has } \\
\text { no effective WT } \\
\text { or IHT. } \\
\text { See capping } \\
\text { mechanism in } \\
\text { liquidity column. }\end{array}$ & $\begin{array}{l}\text { Self-assessment } \\
\text { valuation on } 31 \\
\text { December every year. } \\
\text { Separate WT form filed } \\
\text { every year in June. } \\
\text { Avoidance involving } \\
\text { splitting assets, using } \\
\text { exemption on business } \\
\text { assets; moving to Madrid } \\
\text { where } 100 \% \text { deduction. } \\
\text { Capping mechanism can } \\
\text { be manipulated by those } \\
\text { who can control their } \\
\text { income and thus reduce } \\
\text { WT. Harder for those on } \\
\text { a high salary. }\end{array}$ & $\begin{array}{l}2019 \text { - } € 1.1 \text { billion in } \\
\text { revenue. } \\
\text { Exempt minimum } \\
\text { threshold is } € 700,000 \\
\text { per individual with an } \\
\text { additional } € 300,000 \\
\text { exemption on the main } \\
\text { residence - so a couple } \\
\text { may have up to } € 2 \\
\text { million exemption. } \\
\text { Some regions such as } \\
\text { Catalonia have only } \\
€ 500,000 \text { exemption } \\
\text { per individual. } \\
\text { However, some } \\
\text { regions, e.g. Madrid, } \\
\text { have up to } 100 \% \\
\text { deduction and so no } \\
\text { wealth tax and there is } \\
\text { large regional latitude. } \\
\text { Rates set nationally at } \\
\text { between } 0.20 \% \text { to } \\
2.50 \% \text { above } € 10.69 \\
\text { million but some } \\
\text { regions have higher } \\
\text { rateses e.g. Balearic } \\
\text { Islands. } \\
\text { No cap by reference to } \\
\text { overall wealth but } \\
\text { some cap by reference } \\
\text { to income - see } \\
\text { liquiditity column }\end{array}$ \\
\hline
\end{tabular}




\begin{tabular}{|c|c|c|c|c|c|c|c|c|c|}
\hline Switzerland & $\begin{array}{l}\text { Cantonallly-based from } \\
1848 \text {. Federal only had } \\
\text { control of indirect } \\
\text { taxes. Cantons levied } \\
\text { income tax and wealth } \\
\text { tax. In } 1915 \text { Swiss } \\
\text { Confederation given } \\
\text { power to levy one time } \\
\text { war taxes - WT up to } \\
0.15 \% \text { Federal WT } \\
\text { abolished in } 1959 \text { for } \\
\text { individuals Cantons } \\
\text { continued to levy } \\
\text { wealth taxes. } \\
\text { All } 26 \text { Cantons levy a } \\
\text { wealth tax but at } \\
\text { different rates and } \\
\text { exemptions. } \\
\text { Some calls for Swiss } \\
\text { WT to be abolished } \\
\text { and replaced by CGT } \\
\text { which currently does } \\
\text { not operate in } \\
\text { Switzerland. }\end{array}$ & $\begin{array}{l}\text { All assets, real } \\
\text { estate, private } \\
\text { residences. } \\
\text { All securities, } \\
\text { works of art, } \\
\text { jewellery, but } \\
\text { not personal } \\
\text { household items } \\
\text { and claims on } \\
\text { pension fund } \\
\text { payments. } \\
\text { Excludes foreign } \\
\text { businesses or } \\
\text { permanent } \\
\text { establishments } \\
\text { and real estate } \\
\text { situated abroad } \\
\text { although taken } \\
\text { into account for } \\
\text { determining } \\
\text { rate. }\end{array}$ & $\begin{array}{l}\text { Married } \\
\text { couples - } \\
\text { wealth added } \\
\text { together to } \\
\text { determine tax } \\
\text { rate. } \\
\text { Geneva does } \\
\text { not } \\
\text { distinguish } \\
\text { between } \\
\text { single or } \\
\text { married } \\
\text { taxpayers but } \\
\text { Zurich does } \\
\text { and gives an } \\
\text { exempt } \\
\text { threshold. } \\
\text { Minor } \\
\text { children's } \\
\text { assets are } \\
\text { included in } \\
\text { the parents' } \\
\text { wealth. }\end{array}$ & $\begin{array}{l}\text { Swiss residents } \\
\text { subject to } \\
\text { wealth tax on } \\
\text { worldwide } \\
\text { assets. No exit } \\
\text { charge but } \\
\text { prorated if } \\
\text { leave or arrive } \\
\text { half way } \\
\text { through the } \\
\text { year. } \\
\text { No WT if forfait } \\
\text { operates but } \\
\text { otherwise all } \\
\text { Swiss residents } \\
\text { subject to WT. } \\
\text { Non-residents } \\
\text { pay on Swiss } \\
\text { real estate and } \\
\text { Swiss } \\
\text { businesses or } \\
\text { PEs but not on } \\
\text { Swiss bank } \\
\text { accounts. } \\
\text { Treaty relief in } \\
63 \text { treaties }\end{array}$ & $\begin{array}{l}\text { Revocable trusts treated } \\
\text { as transparent to the } \\
\text { settlor. } \\
\text { Irrevocable } \\
\text { discretionary trusts } \\
\text { settled by Swiss resident } \\
\text { settlor are taxed on } \\
\text { settlor while Swiss } \\
\text { resident. } \\
\text { If trust set up when } \\
\text { settlor not Swiss resident } \\
\text { and settlor later } \\
\text { becomes Swiss resident, } \\
\text { trust assets are not } \\
\text { attributed to settlor if } \\
\text { excluded or to the } \\
\text { beneficiaries if no } \\
\text { benefits received. } \\
\text { Irrevocable fixed interest } \\
\text { trust assets e.g. life } \\
\text { interest can be allocated } \\
\text { to the Swiss resident } \\
\text { beneficiary. } \\
\text { Discretionary } \\
\text { trust/deceased settlor } \\
\text { and Swiss resident } \\
\text { beneficiary - beneficiary } \\
\text { taxed only if receives } \\
\text { benefits. } \\
\text { Usufruct - person with } \\
\text { usufructuary/life interest } \\
\text { is taxed. }\end{array}$ & $\begin{array}{l}\text { Non-business assets: } \\
\text { market value. } \\
\text { Real estate - varies per } \\
\text { canton. Some formulaic } \\
\text { principles. } \\
\text { Value of private companies } \\
\text { determined each year by } \\
\text { cantonal tax authorities } \\
\text { based on inter-cantonal } \\
\text { administrative guidelines } \\
\text { and value determined by } \\
\text { formulaic method. } \\
\text { Calculated weighted } \\
\text { average of its earnings } \\
\text { value and net asset value. } \\
\text { See Swiss paper. }\end{array}$ & $\begin{array}{l}\text { Wealth tax not } \\
\text { credited against } \\
\text { income tax or } \\
\text { deductible. } \\
\\
\text { No capital gains } \\
\text { tax in } \\
\text { Switzerland. } \\
\text { Inheritance and } \\
\text { gift tax levied at } \\
\text { Cantonal level } \\
\text { but usually } \\
\text { minimal. } \\
\text { No deductions } \\
\text { given against } \\
\text { WT. } \\
\text { Note the tax } \\
\text { shield that } \\
\text { operates in } \\
\text { Geneva which } \\
\text { limits combined } \\
\text { tax burden of } \\
\text { income tax and } \\
\text { capital tax to } \\
60 \% \text { of net } \\
\text { taxable income. }\end{array}$ & $\begin{array}{l}\text { Annual return. } \\
\text { Taxpayer files a } \\
\text { combined income and } \\
\text { wealth tax declaration. } \\
\text { Some disputes over } \\
\text { valuation e.g. of private } \\
\text { companies, art, } \\
\text { household items. }\end{array}$ & $\begin{array}{l}\text { Varies between } 0.3 \% \\
\text { and } 1 \% \text { - highest rate is } \\
\text { in Geneva. } \\
\text { Tax-free threshold } \\
\text { around US } \$ 50,370 \text { to } \\
\text { US } \$ 251,856 \text { and } \\
\text { generates } 3.8 \% \text { of total } \\
\text { tax revenue. } \\
\text { Significant differences } \\
\text { between cantons. }\end{array}$ \\
\hline
\end{tabular}




\section{References}

Banoun, B. (2020). Wealth tax: Norway. Wealth Tax Commission Background Paper, 138.

Dupas, M. (2020). Wealth Tax: France. Wealth Tax Commission Background Paper, 134.

Eckert, J-B., and Aebi, L. (2020). Wealth Taxation in Switzerland. Wealth Tax Commission Background Paper, 133.

Paoletto, A., Monte, M., and Bonomi, R. (2020). Wealth tax: Italy. Wealth Tax Commission Background Paper, 136.

Ramallo, A. (2020). Wealth Tax: Spain. Wealth Tax Commission Background Paper, 132.

Rehr, R. (2020). Financing COVID-19 costs in Germany: is a wealth tax a sensible approach? Wealth Tax Commission Background Paper, 131.

Tirard, J-M. (2020). Wealth Taxes in France. Wealth Tax Commission Background Paper, 135.

Vanvari, G. and Ta, K. (2020). Wealth tax: India. Wealth Tax Commission Background Paper, 137. 